Hautarzt 2009 60 :376-377

DOI 10.1007/s00105-009-1760-y

๑) Springer Medizin Verlag 2009

\author{
D. Bruch-Gerharz ${ }^{1}$ T. Ruzicka ${ }^{2}$ \\ ${ }^{1}$ Hautklinik des Universitätsklinikums Düsseldorf, Heinrich-Heine-Universität \\ 2 Klinik und Poliklinik für Dermatologie und Allergologie, \\ Ludwig-Maximilians-Universität München
}

\title{
Pädiatrische Dermatologie
}

Teil II

Hautkrankheiten im Kindesalter spielen im Klinik- und Praxisalltag sowohl des Dermatologen als auch des Pädiaters eine große Rolle. Mithin steigt das Interesse an der Kinderdermatologie - eine Tatsache, die sich beispielhaft darin widerspiegelt, dass die renommierte Monatsschrift Kinderheilkunde dem Leitthema Hautkrankheiten im Februar 2008 eine umfassende Ausgabe widmete. So weist der Herausgeber Prof. Dietrich Reinhardt, Direktor des Dr. von Haunerschen Kinderspitals der Ludwig-Maximilians-Universität München, ausdrücklich auf die Bedeutung des engen interdisziplinären Kontaktes zwischen beiden Fachgebieten und der Notwendigkeit der Bündelung ihrer Kompetenzen hin. Auch das vorliegende Leitthemenheft „Pädiatrische Dermatologie Teil II“ wendet sich folglich nicht nur an interessierte Hautärzte, sondern auch an Pädiater und alle anderen klinisch tätigen Ärzte, die Kinder betreuen.

Im ersten Beitrag des vorliegenden Leitthemenheftes zeigen M. Laimer und Koautoren auf, welche Bedeutung die molekularbiologische Forschung bei der Aufklärung verschiedener Genotypen der Epidermolysis bullosa hereditaria gespielt hat. So ist die Verletzlichkeit der Haut und Schleimhäute mit nachfolgender Blasenbildung durch zahlreiche Mutationen in Genen bedingt, die für Strukturproteine der Haut kodieren. Hierdurch wurde eine neue Klassifikation der Epidermolysis bullosa hereditaria mit ihren verschiedenen Phänotypen ermöglicht, die für die Prognose und Therapie von entscheidender Bedeutung sind.
Der sich anschließende Beitrag hebt hervor, dass Pilzinfektionen der Haut und hautnahen Schleimhäute in ihrer Bedeutung oft unterschätzt werden, obwohl sie in ihrer Zahl und Dauer alle anderen mikrobiellen Infektionen bei Weitem übertreffen und gerade im Kindesalter in ihrer Häufigkeit zunehmen. Dies unterstreicht die Notwendigkeit, diese Infektionskrankheiten wieder konsequenter in die differenzialdiagnostischen Überlegungen einzubeziehen. Eine sorgfältige Diagnostik einschließlich eines mykologischen Erregernachweises mittels Mikroskopie am nativen Direktpräparat und kultureller Anzüchtung schafft die Voraussetzung für eine effektive Therapie. Stets ist auch die konsequente Elimination von Prädispositionsfaktoren unverzichtbar (D. BruchGerharz und T. Ruzicka).

\section{$>$ Pilzinfektionen der Haut und hautnahen Schleimhäute übertreffen in Zahl und Dauer alle anderen mikrobiellen Infektionen und nehmen gerade im Kindesalter deutlich zu}

Ektoparasitosen gehören ebenfalls zu den häufigen Dermatosen im Kindesalter und bieten morphologisch ein sehr breites Reaktionsspektrum, das u. a. Papeln, Vesikel, Blasen, Urtikae, ekzematöse Läsionen und Exkoriationen umfasst. Dabei sind neben permanent auf oder in der Haut lebenden Ektoparasiten (Krätzemilben, Kopfläuse) auch temporäre (Wanzen, Flöhe) oder akzidentelle (Erntemilben, Zerkarien) Ek- toparasiten, für die der Mensch eigentlich einen Fehlwirt darstellt, für Erkrankungen und Epidemien im Kindesalter verantwortlich. Die Besonderheiten der Epidemiologie, Klinik, Diagnostik und Therapie der kindlichen Ektoparasitosen werden im Beitrag von R. Fölster-Holst und H. Hamm eingehend beschrieben.

Im letzten Beitrag zum Schwerpunktthema wird schließlich die häufigste Hautkrankheit der Jugendlichen, die Acne juvenilis, abgehandelt. Fast jeder Jugendliche ist in der Pubertät von Akne in unterschiedlicher Ausprägung betroffen, und häufig bestehen aufgrund des klinischen Erscheinungsbildes psychische Probleme. Pathogenetische Faktoren umfassen eine androgengesteuerte Überproduktion von Talg, eine follikuläre Hyperkeratose, eine mikrobielle Besiedlung sowie immunologische und entzündliche Prozesse. Alle Formen der juvenilen Akne sind heute mit pathogeneseorientierten Therapiestrategien gut kontrollierbar, erfordern aber ein gutes Arzt-Patienten-Verhältnis und Geduld aufgrund der Langwierigkeit der Behandlung (P. Lehmann).

An dieser Stelle möchten wir uns bei allen Autoren und Koautoren des vorliegenden Leitthemenheftes für ihren Beitrag zu einer praxisnahen Fort- und Weiterbildung auf dem wichtigen Gebiet der pädiatrischen Dermatologie bedanken. Unser besonderer Dank gilt aber auch den Lesern des im März 2009 erschienenen ersten Teils des Leitthemas „Pädiatrische Dermatologie“ für die große Resonanz, die wir erfahren haben. 
Wir hoffen, dass der zweite Teil ähnliches Interesse hervorruft und dass unsere Kollegen von den hier vorgestellten Übersichtsbeiträgen in ihrer täglichen Arbeit profitieren.

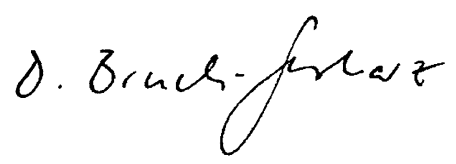

Daniela Bruch-Gerharz

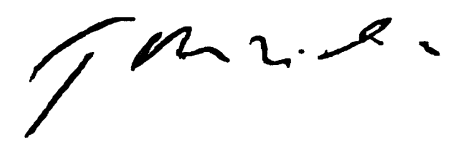

Thomas Ruzicka

\section{Korrespondenzadressen}

\section{Prof. Dr. D. Bruch-Gerharz}

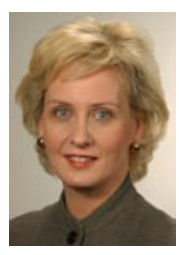

Hautklinik des Universitäts-

klinikums Düsseldorf,

Heinrich-Heine-Universität

Moorenstraße 5,

40225 Düsseldorf

Bruch-Gerharz@med.

uni-duesseldorf.de

\section{Prof. Dr. Dr. h.c. T. Ruzicka}

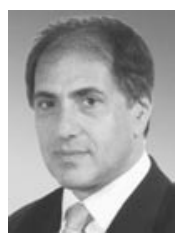

Klinik und Poliklinik für Dermatologie und Allergologie, Ludwig-Maximilians-Universität München Frauenlobstraße 9-11, 80337 München Thomas.Ruzicka@med. uni-muenchen.de

\section{Möchten Sie einen Beitrag für „Der Hautarzt" einreichen?}

Wir freuen uns, dass Sie unsere Zeitschrift "Der Hautarzt" mitgestalten möchten. Um Ihnen bei der Manuskripterstellung behilflich zu sein, haben wir für unsere Autoren Hinweise zusammengestellt, die Sie im Internet finden unter www.DerHautarzt.de (Für Autoren). Bitte senden Sie Ihren fertigen Beitrag an:

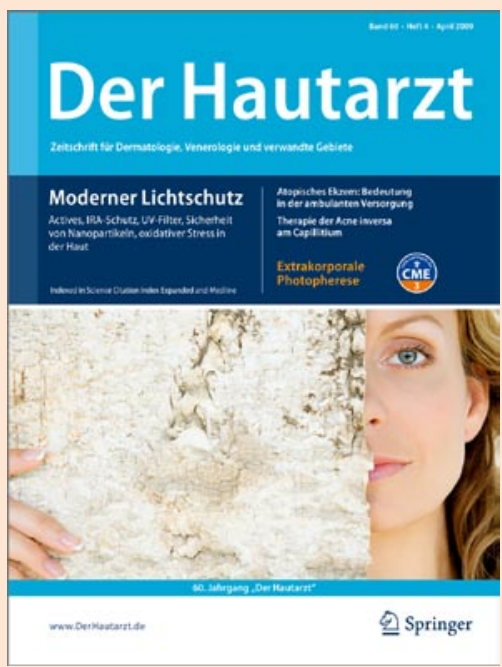

\section{Übersichten:}

Prof. Dr. Alexander Kapp

Klinik und Poliklinik für Dermatologie und Venerologie, Medizinische Hochschule Hannover, Ricklinger Straße 5, 30449 Hannover

Tel: +49-511-9246-232

Fax: +49-511-9246-234

freimooser.martina@mh-hannover.de

Originalien, Kasuistiken:

Prof. Dr. Thomas Ruzicka

Klinik und Poliklinik für Dermatologie, LMU München

Anfragen an:

PD Dr. Roland Kruse

Hautklinik des Universitätsklinikums

Düsseldorf, Moorenstr. 5 ,

40225 Düsseldorf

Tel:+49-211-81-18328

Fax:+49-211-81-04905

s.gehrke@med.uni-duesseldorf.de
Weiterbildung •

Zertifizierte Fortbildung:

Anfragen an:

Prof. Dr. Michael Meurer Klinik und Poliklinik für Dermatologie an der Universitätsklinik Carl Gustav Carus, TU Dresden, Fetscherstr. 74, 01307 Dresden meurer@rcs.urz.tu-dresden.de

Prof. Dr. Rolf-Markus Szeimies Dermatologische Klinik der Universität Regensburg, Franz-Josef-Strauß-Allee 11, 93053 Regensburg Rolf-Markus.Szeimies@ klinik.uniregensburg.de

Wie lautet Ihre Diagnose?: Prof. Dr. Karin Scharffetter-Kochanek Universitätsklinik und Poliklinik für Dermatologie, Abteilung für Dermatologie und Allergologie, Maienweg 12, $89081 \mathrm{UIm}$

Tel: +49-731-500-21801

Fax: +49-731-500-21870

In der Diskussion: Prof. Dr. Alexander Kapp/ Prof. Dr. Thomas Werfel Klinik und Poliklinik für Dermatologie und Venerologie, Medizinische Hochschule Hannover, Ricklinger Straße 5, 30449 Hannover freimooser.martina@mh-hannover.de

Leserforum:

Prof. Dr. Hans F. Merk

Klinik für Dermatologie und

Allergologie, Universitätsklinikum der RWTH, Pauwelsstraße 30, 52057 Aachen Hans.Merk@post.rwth-aachen.de

Neue Therapiekonzepte und Studien: Prof. Dr. Alexander Kapp/

Prof. Dr. Thomas Werfel

Klinik und Poliklinik für

Dermatologie und Venerologie, Medizinische Hochschule Hannover, Ricklinger Straße 5, 30449 Hannover freimooser.martina@mh-hannover.de

Gesundheitsökonomie:

Prof. Dr. Michael Jünger

Klinik und Poliklinik für Hautkrankheiten, Klinikum der Ernst-MoritzArndt-Universität Greifswald, Fleischmannstraße 42-44, 17487 Greifswald juenger@uni-greifswald.de 